\title{
An Energy-Saving Embedded Computer Hosiery Machine Control System
}

\author{
Chong Chen, Simin Peng, Cuifeng Shen, Xiaohui Duan \\ School of Electrical Engineering, Yancheng Institute of Technology, PR China.
}

\begin{abstract}
Performances requirements of computer hosiery machine control system is analyzed, and the system scheme of double core processors cooperation control, which respectively accomplish human-computer interaction and control function, is designed. Aiming at the main electrical control points in computer hosiery machine, the system hardware circuit and control methods of various field devices were given. The results show that, the system control the objects through changing the frequency of electrical signal, and it has the characteristics of safe and power-saved.
\end{abstract}

Keywords: energy-saving; computer hosiery machine; embedded system; double core processors

\section{INTRODUCTION}

Socks, as one of the representative textile product, is necessary for people. They are braided by hosiery machine. The hosiery machine is the main types of braiding cotton hoses and stockings. Computer hosiery machine is a highly automated knitting equipment, integrated computer technology, electronic technique, knitting technology and machining technique. Foreign advanced hosiery machine manufacturer devoted to the technological innovation and product upgrade all the time, constantly pushing forward computer hosiery machine development technology ${ }^{[1][2]}$. However, when foreign high-grade hosiery machines are always sold to our country with whole machine, without providing control system. Therefore, research and develop computer hosiery machine control system having self-owned intellectual property is becoming very urgent.

There are many field devices need to control in hosiery machine. If the normally open and close method is adopted, leading to very big current and violent change of peak current, which not only waste a lot of power, but also easy to heat and burn out the components. Therefore proper control method is always needed, fitted with reliable hardware circuit and software algorithm, finally forming a steady and power-saved hosiery machine control system. If variable frequency control idea is applied into AC servo motor, stepper motor, electromagnetic valve, needle selector and other field devices, it can better resolve the problem of softly starting and stopping of hosiery machine control system, greatly decreasing the current of the system, thus the heat dissipating capacity and energy consumption is reduced. In this paper, safe and power-saved computer hosiery machine control system is designed. Various of field devices are better controlled, with the function of receiving the fabric data of upper computer and braiding corresponding flower color socks.

\section{InTegral Design Scheme of Computer Hosiery Machine Control System}

\subsection{Performance Requirements}

Computer hosiery machine control system including drive control system, jacquard system, manmachine interface and fault detection. Its main work is shown in FIgure1. 


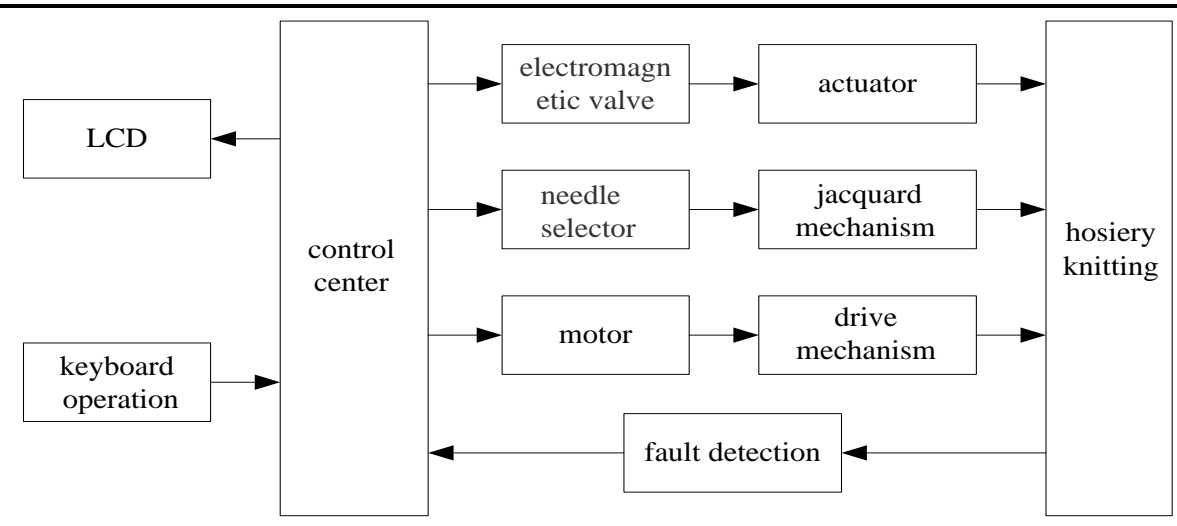

Figure1. Overall framework of Computer hosiery machine control system

As a staple and speedy computer hosiery machine control system, its controller should meet the following requirements: a. Strong computational capability and real-time control. There are many control points and multiple fault detection. These control points respond quickly when the controller send instructions, especially servo motor, because not only the angle of rotation need to control quickly and accurately, but also need to detect its location. b. Convenient keyboard operation and human-computer interaction interface. During the process of knitting, the operator can realize the start and stop of computer hosiery machine, set the process parameters, and test the mechanical components independently. Meanwhile the display module can display the process parameters and the current state of computer hosiery machine. c. Fault detection and process function. During the process of knitting, there are some mechanical faults. The controller should detect them timely, stop the computer hosiery machine and then display the fault causes through the display module.

\subsection{Overall Design of the System}

The computer hosiery machine control system based on dual core processor mode is shown in Figure.2. The whole system including dual core processor control system composed of humancomputer interactive processor and main controller, and remote monitoring and control system using wire and wireless communication. The function of human-computer interaction is realized with the embedded microprocessor as the core. The jump of LCD interface is realized by key, so as to inquire related information and function. The mechanical drive control is realized with the digital signal processor as the core. It is primarily responsible for the coordinate operation of each module of hosiery machine and control the field devices. The main function module including needle selector module, electromagnetic valve module, stepper motor module and servo motor module. The control system realize the real-time control of every field device through the output of control signal and the collection of corresponding feedback signal, finally complete all weaving movements. Wire and wireless communication are adopted to realize the remote control of pattern digital file, total socks and speed.

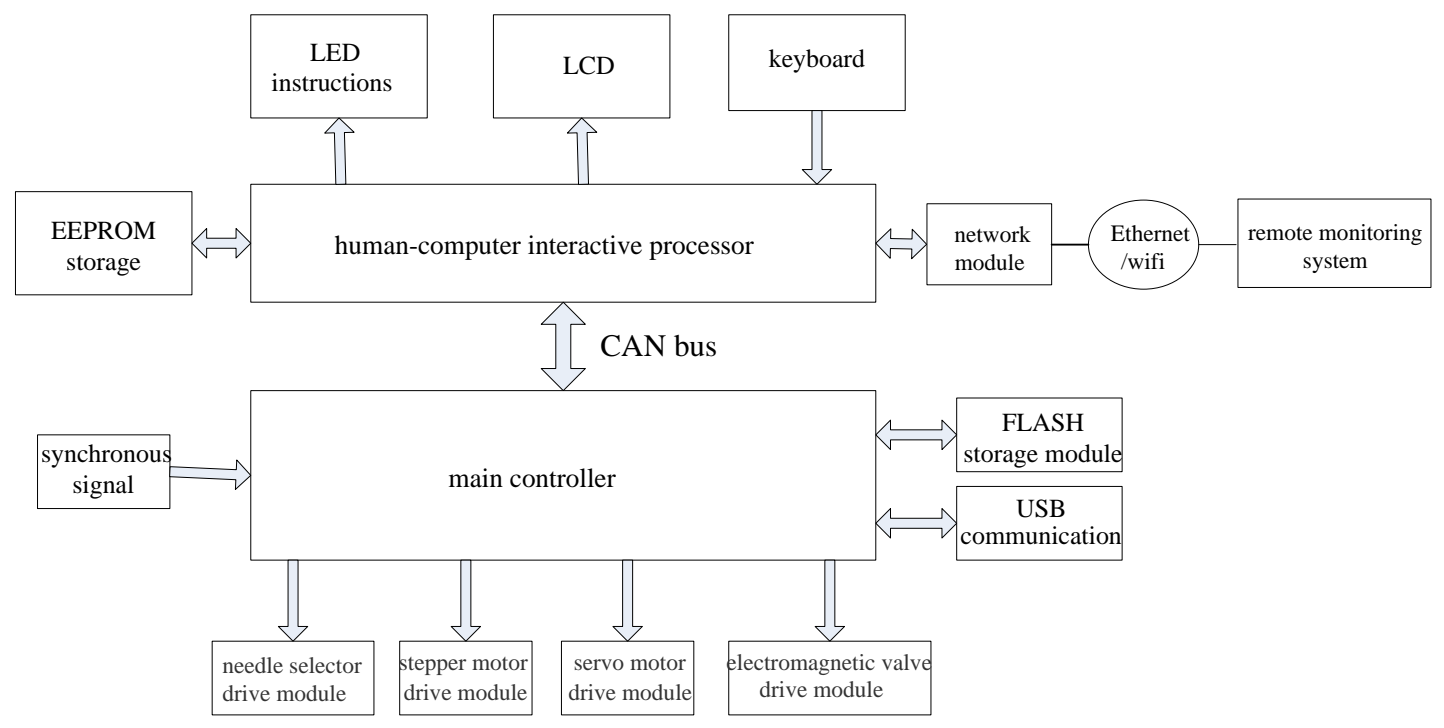

Figure2. Scheme of hosiery machine control system based on dual core processor mode 


\section{HARDWARE DESIGN OF THE SYSTEM}

\subsection{Electromagnetic Valve Drive Module}

Electromagnetic valve is equivalent to a switch device, and it only need the controller send ou two states to control it power on and power off. In this system, the driving voltage of electromagnetic valve is $24 \mathrm{~V}$, but the control signal from control terminal is only $5 \mathrm{~V}$. Therefore, darlington transistor is added in the hardware system, making the $5 \mathrm{~V}$ control signal convert to $24 \mathrm{~V}$ drive signal. The hardware circuit of electromagnetic valve is shown in Figure.3.

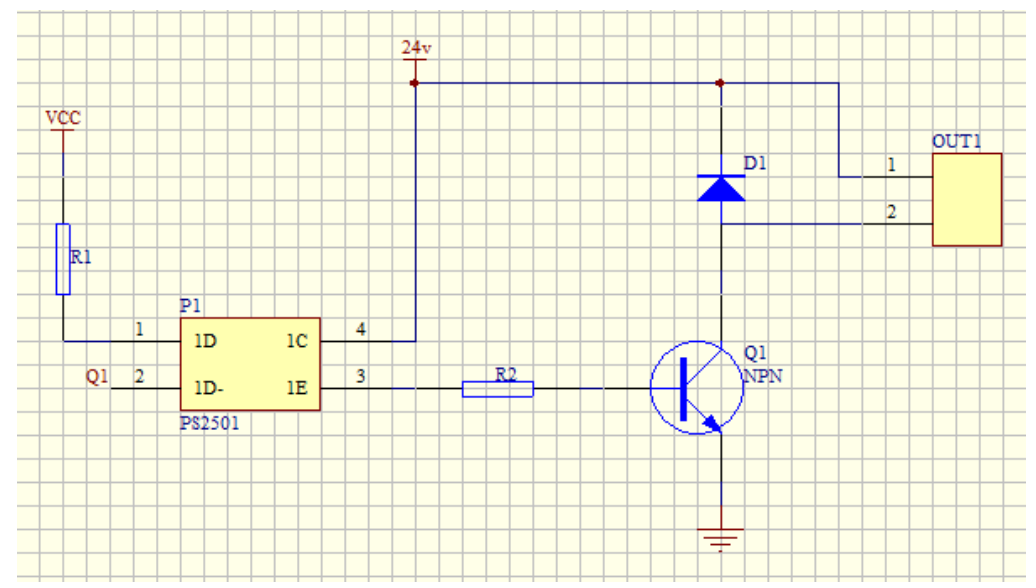

Figure3. Hardware control circuit of electromagnetic valve

In order to reduce the interference of the large current on the $24 \mathrm{~V}$ drive voltage, the photo-coupler is adopted to isolate, so that both ends of the signal is basically not affected by each other. In Figure.3, O1 is the control signal send out by main controller, and OUT1 will be connected to the controlled object electromagnetic valve.

\subsection{Needle Selector Drive Module}

The needle selector adopted in the system needs at least $24 \mathrm{~V}$ drive voltage, and it should change in both positive and negative direction. The violent current change will have a big disturbance on the data signal of control end. As can be seen from Figure.4, when Q1A turns on and Q1B turns off, OUT1A out pus high-level. While Q1A turns off and Q1B turns on, OUT1A out pus low-level. Similarly, OUT1B can also outputs high-level or low-level, by control the turn-on and turn-off state of Q1C and Q1D. In Figure.4, OUT1 A and OUT1B will add to the two ends of needle selector. When OUT1A out pus high-level and OUT1B out pus low-level, this positive voltage can realize the forward operation of needle selector, otherwise back operation therefore, the different control signal sends out by control end can change the voltage direction added in the two ends of needle selector, thus realize the corresponding operation.

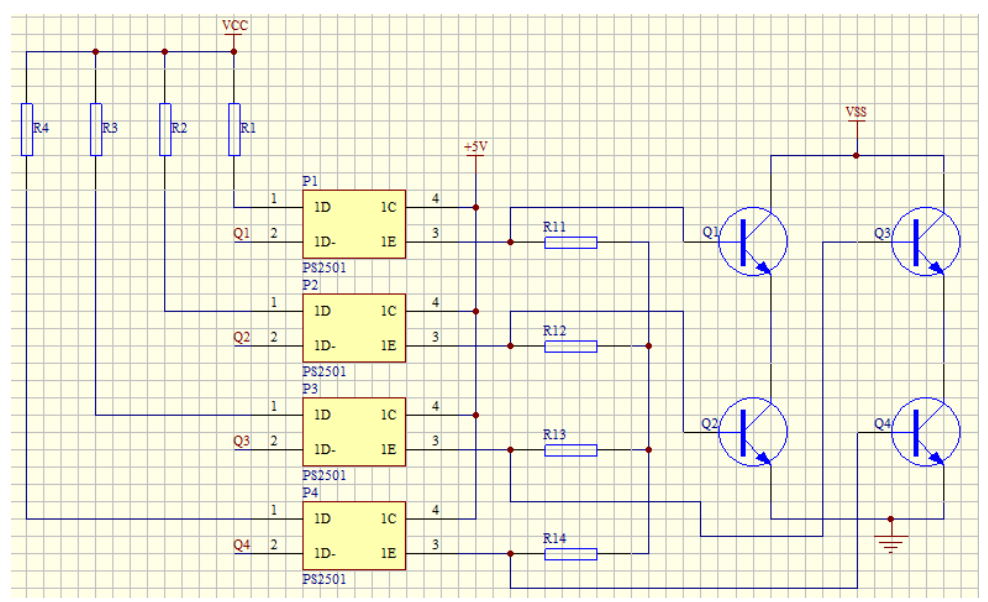

Figure4. Hardware drive circuit of needle selector

\subsection{Stepper Motor Drive Module}

Two phase hybrid stepper motor is adopted in this paper, and its drive circuit is shown in Figure.5. 


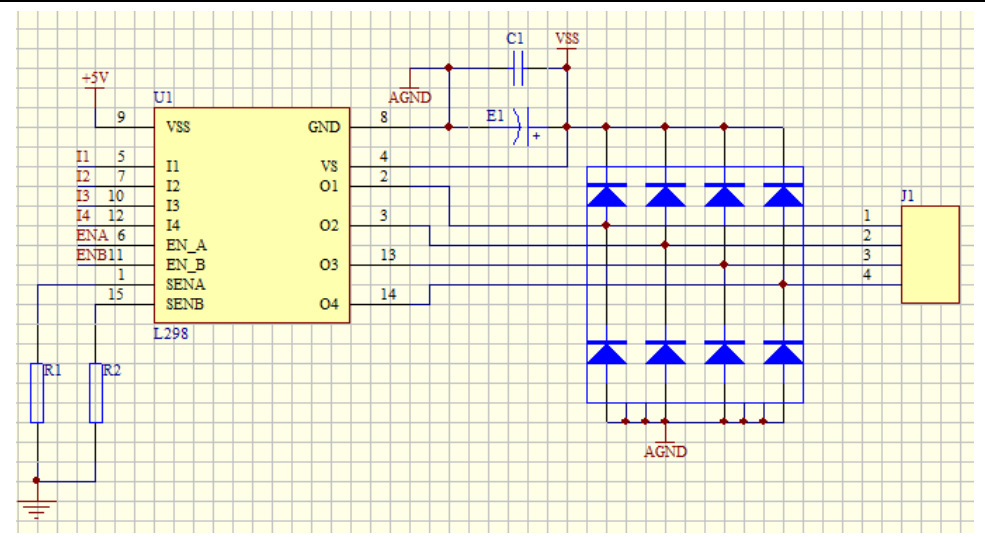

Figure5. Hardware drive circuit of stepper motor

The stepper motor rotates according to the change of phase signal, realizing the function of yarn delivery. In the operation process of hosiery machine, the speed of the stepper motor needs to be changed according to the operation of the other parts, so as to ensure the smooth transmission of the yarn. Variable frequency control technology is adopted in this paper, through changing the frequency of the phase signal in real time to control the speed and position of the stepper motor.

L298 is a dual $\mathrm{H}$ bridge chip, which can directly drive a one phase stepper motor. A $0.5 \Omega$ Precision resistor is connected in series at the ground end of each $\mathrm{H}$ bridge, achieving real-time the current value of the bridge. When the current is too large, the entire module should be closed immediately. L6210 is a diode array, which plays the role of after flow, realizing the fast switching of phase signal.

\subsection{Servo Motor Hardware Circuit Structure}

Three phase AC servo motor is adopted to drive the spindle rotating. Control block diagram is shown in figure.6. AC-DC-AC voltage source inverter circuit, which mainly include main circuit and control circuit, is adopted. Three phase AC electricity is firstly dealt with rectifier and filter capacitor, and then with pump voltage suppression circuit and power inverter, then drive permanent magnet synchronous motor. Control circuit takes DSP as the core, and it is composed of voltage and current detection circuit, motor rotor speed, displacement detection and drive protection circuit.

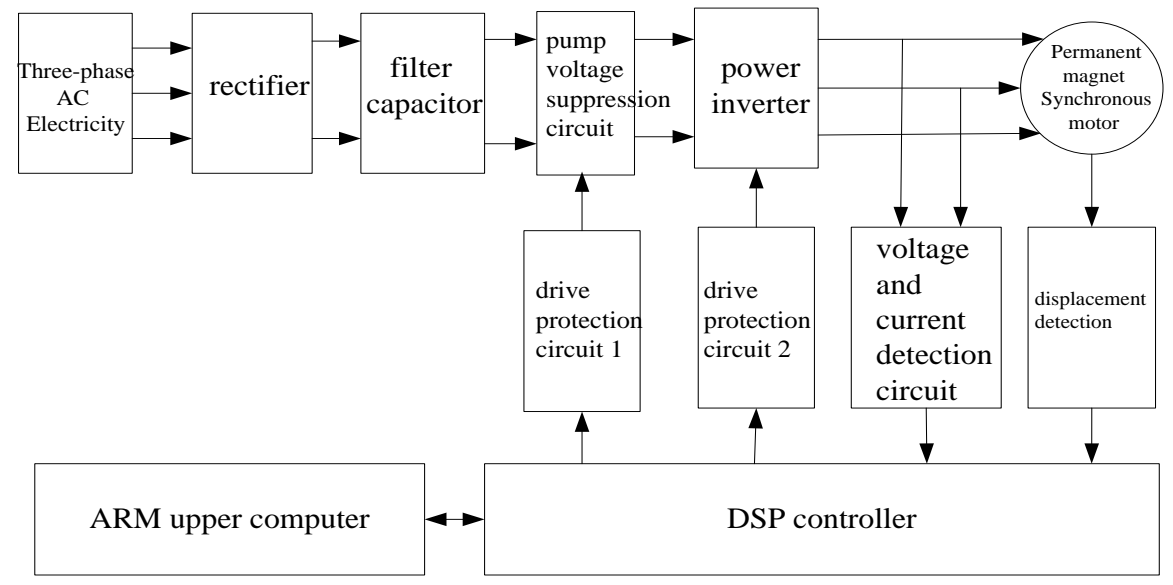

Figure6. Hardware control block diagram of servo motor

\section{Frequency Control Method of Field Device}

\subsection{Electromagnetic Valve Control}

Interval power method is adopted, that is, the electromagnetic valve is electrified for a certain time, then the voltage is withdrawn. And give it power again before exit, so as repeated interval, forming PWM wave with a certain period and duty ratio. This control signal is adopted to substitute DC voltage signal to control electromagnetic valve, as is shown in Fig.7. 


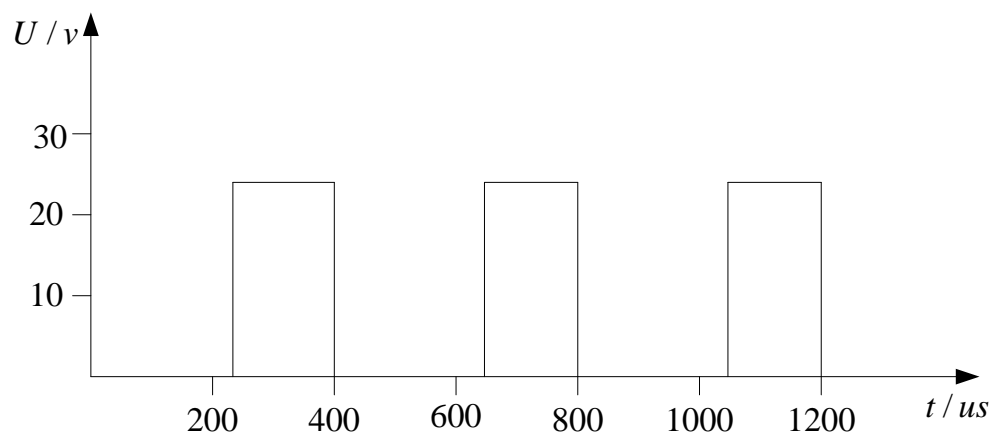

Figure7. Drive voltage signal of electromagnetic valve with interval power

\subsection{Needle Selector Control}

Needle selector work through changing the current direction of electrifying coil. In theory, there are three states of needle selector. The positive voltage applied on the electrifying coil, then needle selection knife enter. While the reversed voltage applied on the electrifying coil, then needle selection knife exit. And if the coil is not electrified, it keep current state. In this paper, the control mode of theoretical power plus interval power is adopted, which through decreasing the total conduction time to resolve the heating problem. Driving voltage signal wave is shown in Fig.8. That is, interval power is adopted when the needle selection knife enter, and theoretical power is adopted when the needle selection knife exit. It not only resolve the over-hot problem of needle selector with over-long power on, but also make the needle selection knife operate timely and steadily.

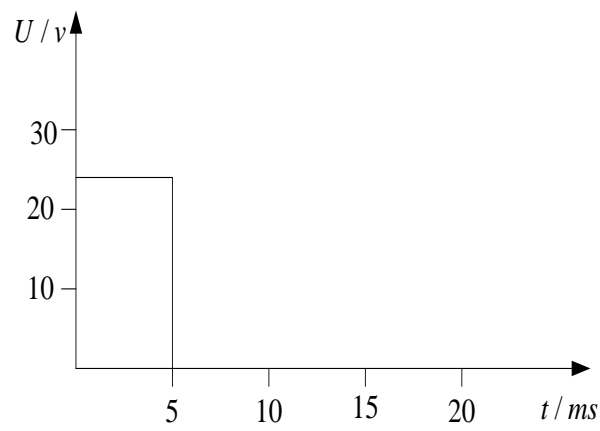

(a)theoretical power mode

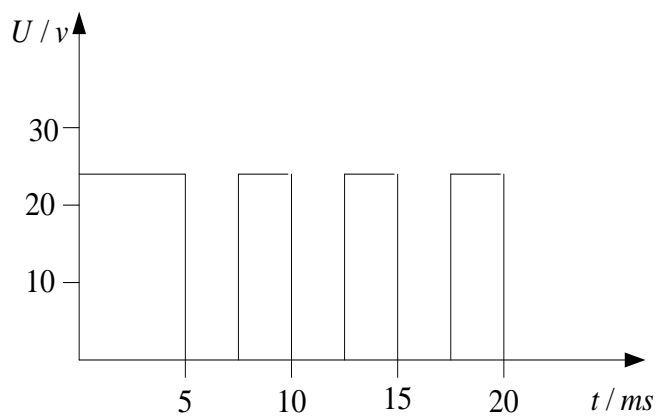

(b)interval power

Figure8. Drive voltage signal of needle selector under two power mode

\subsection{Stepper Motor Control}

There are three working modes of stepper motor: half step, two-phase incentive basic step accuracy, and one-phase incentive basic step accuracy. The second working mode is adopted. When the hosiery machine is running, aiming at different process links, the running speed of stepper motor is different. Variable frequency control technology is adopted, gradually changing the frequency of phase signal through software, and finally making the motor switching speed safely.

\subsection{Servo Motor Control}

Three-phase AC permanent magnet synchronous servo motor is selected in this paper. Frequency vector control based on SVPWM is adopted to control the speed, rotation and position.

The servo control system of hosiery machine can improve the performance of traditional hosiery machine DC motor plus mechanical structure, simplify cylinder reciprocating motion, making maintenance and transform socks style simple, and shortening the time of braiding socks. As a result of adopting frequency conversion technology, the variable speed energy saving of servo motor can be realized easily. 


\section{SySTEM DebugGing}

The system includes upper computer and lower computer two parts. Upper computer are pattern design software and pattern file transmission module. Lower computer include pattern file storage module, needle selector, electromagnetic valve and other actuators. Pattern files designed by upper computer are transmitted to hosiery machine control system through USB transmission mode.

\section{CONCLuSiON}

Variable frequency technology is applied into the control of various of hosiery machine field devices, such as electromagnetic valve, needle selector, stepper motor and servo motor, so as to reduce the power and heat dissipating capacity, and construct a safe and power-saved computer hosiery machine control system. After a long time running and experiment, research data results show that the technology theme is feasible.

\section{ACKNOWLEDGMENTS}

This work was supported in part by the National Natural Science Foundation of China under Grant 51507150 and by the Jiangsu Province Industry-University-Research Collaboration Project under BY2016065-08.

\section{REFERENCE}

[1] New developments in the hosiery industry [J]. Melliand Textilberichte, vol.87, no.10, p721-722, October 2006.

[2] Catarino A., Rocha A., Monteiro J.L., Soares F. A Pattern recognition system based on cluster and discriminant analysis for fault identification during production [J]. IEEE International Symposium on Industrial Electronics, p298-303, 2007.

[3] Liu Chang-zhu. Research of the servo control system in computer hosiery machine. Computer technology and its application development. 2009 994-998

[4] Zhu jianjiang, Yu Shiming, Linxin. Research on NAND FLASH in jacquard system of embedded hosiery machine and its application. Computer applications and software.vol.29,no.10, Oct.2012.

[5] Zhangming, Yu Shiming, Lin Zhongneng. Computer hosiery machine controlling system based on frequency convert control. Journal of Mechanical\& Electrical Engineering. Vol. 27 No. 3, Mar. 2010

\section{AUTHORS' BIOGRAPHY}

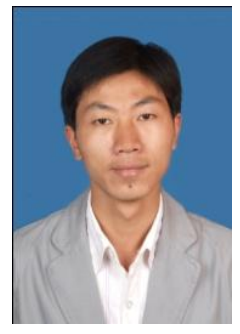

Chong Chen, received his master degree in Electrical Engineering from Jiangsu University in 2008. He is currently at School of Electrical Engineering, YanCheng Institute of Technology, China. His current research interest includes motion control and power electron application.

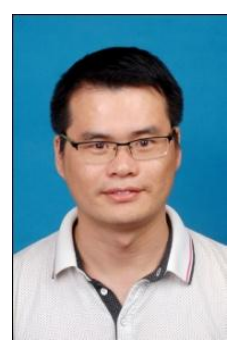

Simin Peng, was born in Hunan province, China, in 1980. He received the B.S degree in automation from Xiangtan University, Xiangtan, China, in 2003, and M.S. degree in 2008, and Ph.D degree in electrical engineering from Shanghai Jiao Tong University in 2013. He is currently working at Yancheng Institute of Technology. His research interests include wind power, micro grid, battery energy storage system and battery manage system. 